A. K. Dupree and A. O. Benz, Eds.

\title{
Periodicities of Radio Flares in RS CVn and Algol Binaries
}

\author{
Mercedes T. Richards \\ Penn State University, Department of Astronomy \& Astrophysics, 525 \\ Davey Lab, University Park, PA 16802, USA
}

Elizabeth B. Waltman

Naval Research Laboratory, Code 7214, 4555 Overlook Ave. S.W., Washington, D.C. 20375, USA

Frank D. Ghigo

National Radio Astronomy Obs., P.O. Box 2, Green Bank, WV 24944

Donald St. P. Richards

Penn State University, Department of Statistics, 326 Thomas Building, University Park, PA 16802, USA

\begin{abstract}
Periodicities of radio flaring activity from the Algol systems $\beta$ Per and $\delta \mathrm{Lib}$ and the RS CVn systems V711 Tau and UX Ari were determined from a continuous 5 -year survey. The radio continuum fluxes at $2.3 \mathrm{GHz}$ and $8.3 \mathrm{GHz}$ were monitored with the NRAO-Green Bank Interferometer for 2096 days from 1995 January to 2000 October. The maximum detected flare strengths at $8.3 \mathrm{GHz}$ were $1.17 \mathrm{Jy}$ for $\beta$ Per, $0.034 \mathrm{Jy}$ for $\delta \mathrm{Lib}, 1.44 \mathrm{Jy}$ for V711 Tau, and 0.82 Jy for UX Ari. Power Spectrum Analysis and Phase Dispersion Minimization were used to determine the periodicity of flaring activity in each binary. The strongest periodicities found were 48.9 days for $\beta$ Per, 120.7 days for V711 Tau, and 141.4 days for UX Ari. No significant periodicities were found for $\delta$ Lib. The continuous survey has demonstrated that there are active and quiescent flaring cycles in V711 Tau and $\beta$ Per.
\end{abstract}

\section{Introduction}

The continuous radio flare monitoring survey began in 1995 January and ended in 2000 October, and each object was observed 2-6 times per day. The primary aim of our survey was to derive reliable estimates of the flaring timescales of stars from data collected continuously over a long baseline. Data collected noncontinuously would produce larger errors because of the uncertainty produced by the gaps in the data coverage. The data were collected with the NRAO-Green Bank Interferometer (GBI), which is a two-element system consisting of 85-foot (26 $\mathrm{m})$ paraboloids. The entire survey spanned 2096 days including interruptions because of maintenance runs and temporary closings of the interferometer. Data 
were collected simultaneously at two frequencies: $2.25 \mathrm{GHz}(13.3 \mathrm{~cm}, \mathrm{~S}$ band $)$ and $8.3 \mathrm{GHz}(3.6 \mathrm{~cm}, \mathrm{X}$ band). The flux densities were determined from the average of the left-left and right-right circular polarizations measured on the 2.4 $\mathrm{km}$ baseline.

The 5-year survey produced 14,959 observations of four systems at each frequency. These included 7442 observations of $\beta$ Per, 3125 of V711 Tau, 1859 of $\delta \mathrm{Lib}$, and 2533 of UX Ari at each frequency. This survey represents the longest and most extensive survey of the Algol and RS CVn classes of magneticallyactive binaries (Richards et al. 2003).

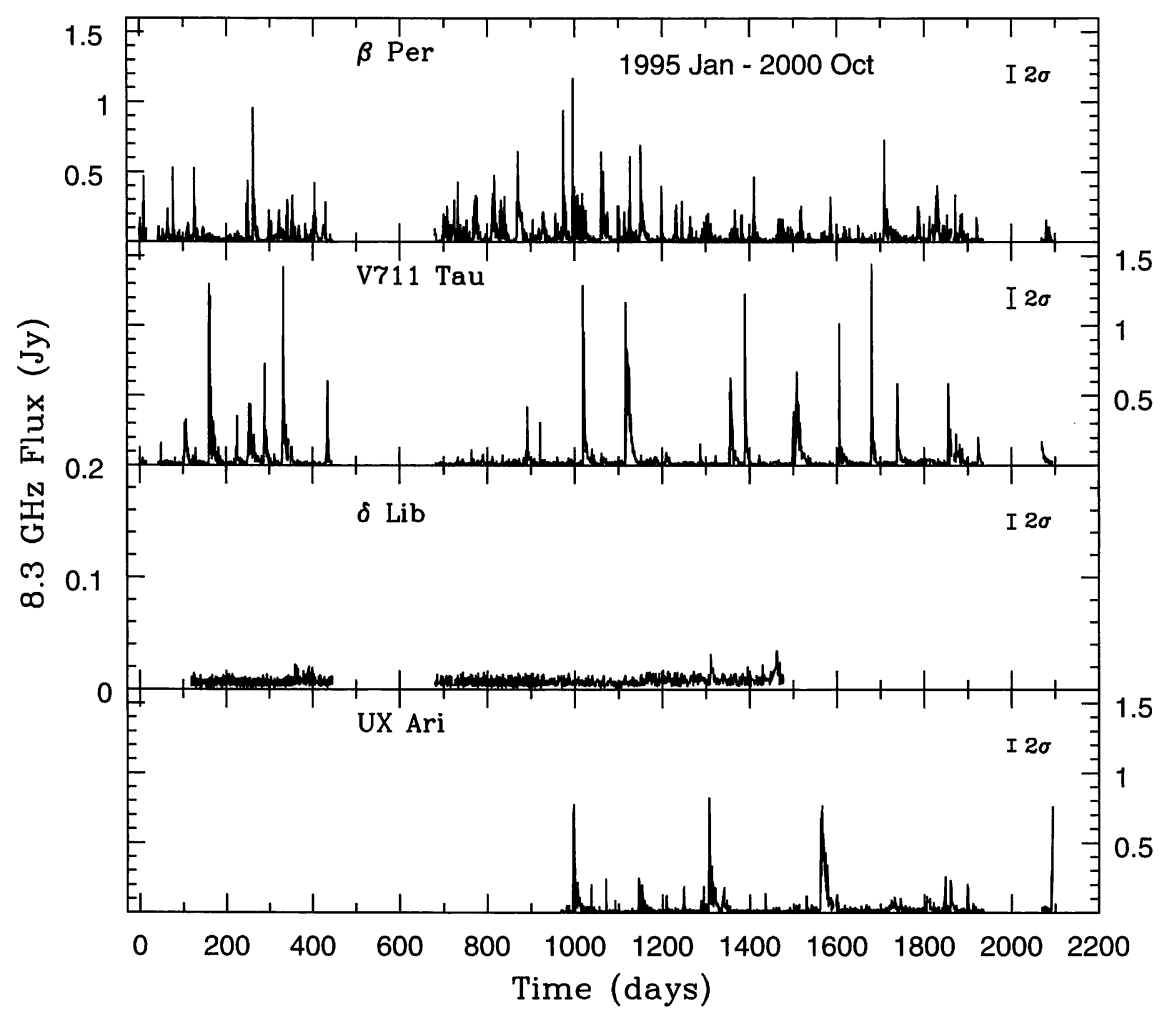

Figure 1. Radio flare data of $\beta$ Per, V711 Tau, $\delta$ Lib, and UX Ari at $8.3 \mathrm{GHz}$ collected from 1995 January 10 to 2000 October 5 . The $2 \sigma$ error bar is shown in the upper right hand corner of each frame. The scale for $\delta$ Lib is enlarged to enhance the weak signal from this binary.

\section{Data Analysis}

Two independent methods were used to determine the flaring periodicities. First, we analyzed the power spectrum obtained by calculating the Fast Fourier transform of the data. The power spectrum will have a sharp peak ( $\delta$-function) at frequencies that correspond to true periodicities in the data (Press et al. 1986). 
Next, we used the Phase Dispersion Minimization (PDM) method (Stellingwerf 1978) in which a test period was chosen and checked to determine if it corresponded to a true periodicity in the data. The goodness-of-fit parameter, $\Theta$, approaches zero when the test period is close to a true periodicity in the data.

The flux density at $2.3 \mathrm{GHz}\left(S_{2.3}\right)$ and $8.3 \mathrm{GHz}\left(S_{8.3}\right)$ and the spectral index, $\alpha$ were analyzed for each system. Here, $S_{\nu_{2}} / S_{\nu_{1}}=\left(\nu_{2} / \nu_{1}\right)^{\alpha}$. The data were analyzed in three groups (see Table 1 ). The two primary targets $\beta$ Per and V711 Tau were monitored during the entire campaign from JD 2449728.6 to JD 2451824.0. Coverage of $\delta$ Lib was from 1995 May 10 UT to 1999 January 28 UT (JD 2449848.5 - JD 2451207.9), while UX Ari was observed from 1997 September 05 UT until the end of the campaign (JD 2450697.9 - JD 2451824.0).

Table 1: Continuous Observing Cycles

\begin{tabular}{cccc}
\hline Dates & JD-2400000 & No. Days & Data Groups \\
\hline 1995 Jan 10 - 1995 Jan 26 & $49728-49744$ & 16 & Group 1 \\
1995 Feb 23 - 1996 Mar 31 & $49772-50174$ & 402 & Group 1 \\
1996 Nov 22 - 2000 Apr 30 & $50410-51665$ & 1255 & Group 2 \\
2000 Sep 11 - 2000 Oct 05 & $51799-51823$ & 25 & - \\
\hline 1995 Jan 10 - 2000 Oct 05 & $49728-51823$ & 2096 & Group 3 \\
\hline
\end{tabular}
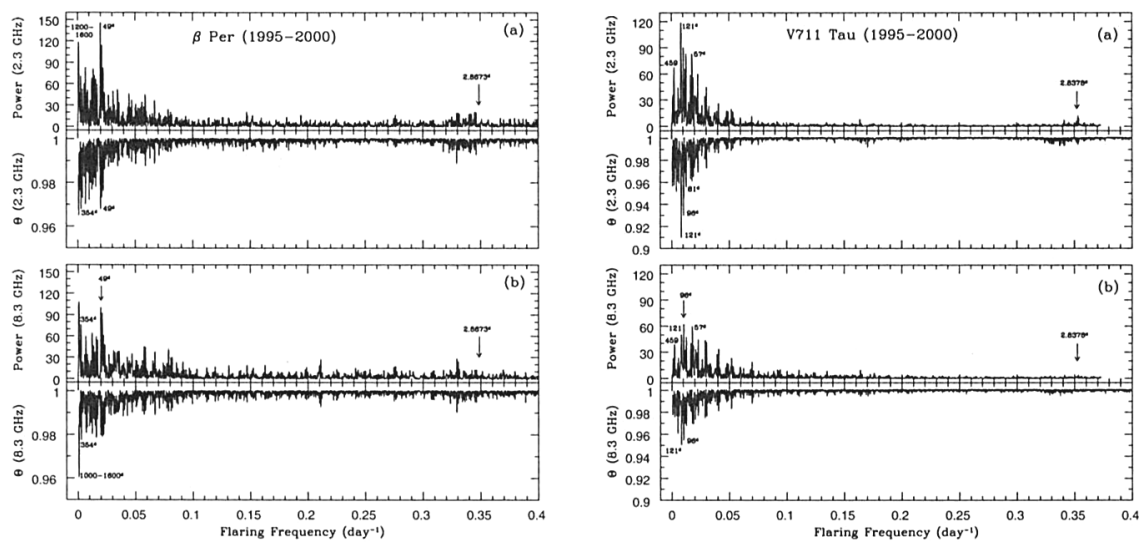

Figure 2. Comparison between the FFT power spectrum and the PDM $\Theta$-statistic for the entire $\beta$ Per and V711 Tau data at (a) 2.3 $\mathrm{GHz}$ and (b) $8.3 \mathrm{GHz}$. The significant periodicities are indicated.

\section{Results}

Continuous, long-term, monitoring of radio flares from two Algol-type binaries ( $\beta$ Per and $\delta \mathrm{Lib}$ ) and two RS CVn binaries (V711 Tau and UX Ari) with the Green Bank Interferometer has provided a useful database for the study of activity cycles. The survey spanned 5.7 years from 1995 January to 2000 October, with continuous monitoring for 4.6 years. Many strong flares were detected with $8.3 \mathrm{GHz}$ fluxes as high as $1.17 \mathrm{Jy}$ in $\beta$ Per, $1.44 \mathrm{Jy}$ in V711 Tau, 

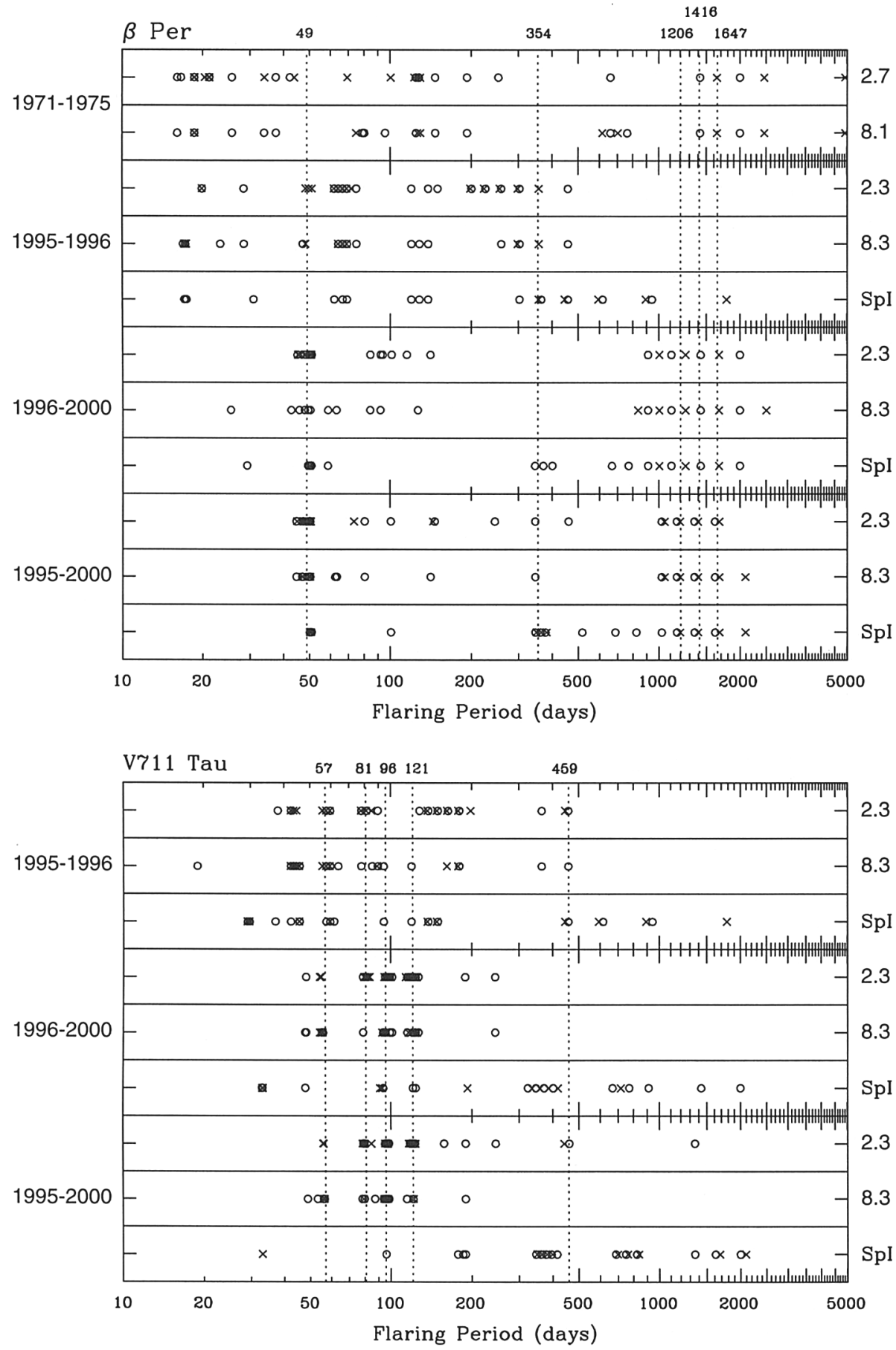

Figure 3. Flaring periods for $\beta$ Per and V711 Tau found from the FFT (x) and PDM (o) techniques for the epochs listed on the left side and for the data sets indicated on the right of each figure. 
and $0.82 \mathrm{Jy}$ in UX Ari. The flux from $\delta \mathrm{Lib}$ was very weak with a maximum $8.3 \mathrm{GHz}$ flux of only $0.034 \mathrm{Jy}$. Only two flares were observed from $\delta$ Lib in 1859 days.

Power Spectrum analysis and Phase Dispersion Minimization were used to identify flaring cycles based on continuous strings of data, as well as the entire data set (Table 1). The 1971 - 1975 Gibson (1976) data of $\beta$ Per at 2.7 and 8.1 GHz were also analyzed. The flaring periods in Figure 3 correspond to the 15 highest periodicities in the power spectrum and the 15 lowest $\Theta$-values. The derived periodicities were checked for correlations with the lowest frequency of the data (corresponding to the time span of the data set), the highest frequency of the data (corresponding to the sampling interval), the Nyquist frequency, or the maximum test period of 2000 days used by the PDM routine.

The primary result of the survey is that flaring cycles are predictable for the systems that have strong flares. The main conclusions were (Richards et al. 2003):

1. Predictable flaring cycles were found for $\beta$ Per, V711 Tau, and UX Ari. Strong flares occur with cycles of $(48.9 \pm 1.7)$ days for $\beta$ Per, $(120.7 \pm 3.4)$ days and $(80.8 \pm 2.5)$ days for V711 Tau, and (141.4 \pm 4.5$)$ days and (52.6 \pm 0.7$)$ days for UX Ari. The $\delta$ Lib data produced inconclusive results. There are strong correlations between the flare cycles and the orbital periods of these binaries.

2. Plots of the flare profiles versus orbital phase show that the strongest flares from the RS CVn binaries tend to occur near $\phi=0.5$ or 1.0 , with more flares visible near $\phi=0.5$ when the more active $\mathrm{K}$ star is toward the observer. In the Algols, the strongest flares were seen near $\phi=0.0$ when the active cooler star was toward the observer.

3. Patterns of alternating active and quiescent flaring activity similar to that seen on the Sun can be identified from the radio flux variations observed in this survey and displayed in Figure 1. These cycles appear to last for more than 500 days in V711 Tau and also in $\beta$ Per. However, our survey could not conclusively determine these long-term cycles.

Acknowledgments. The Green Bank Interferometer (GBI) is a facility of the NSF and was operated during the collection of the data by the NRAO, with funding from the USNO, NRL, NRAO, and NASA High Energy Astrophysics Program. Mercedes Richards was a Guest Observer on the GBI during the first phase of the data collection. This research was supported by AFOSR grants F49620-92-J-0024 and F49620-94-1-0351, NSF grants AST-9618461 and AST0074586, and NASA grant NAG 5-3056.

\section{References}

Gibson, D. 1976, Ph.D. thesis, University of Virginia

Press, W. H., Flannery, B. P., Teukolsky, S. A., \& Vetterling, W. T. 1986, Numerical Recipes (Cambridge: Cambridge University Press)

Richards, M. T., Waltman, E. B., Ghigo, F. D., \& Richards, D. St. P. 2003, ApJS, 147, 337

Stellingwerf, R. F. 1989, AJ, 224, 953 


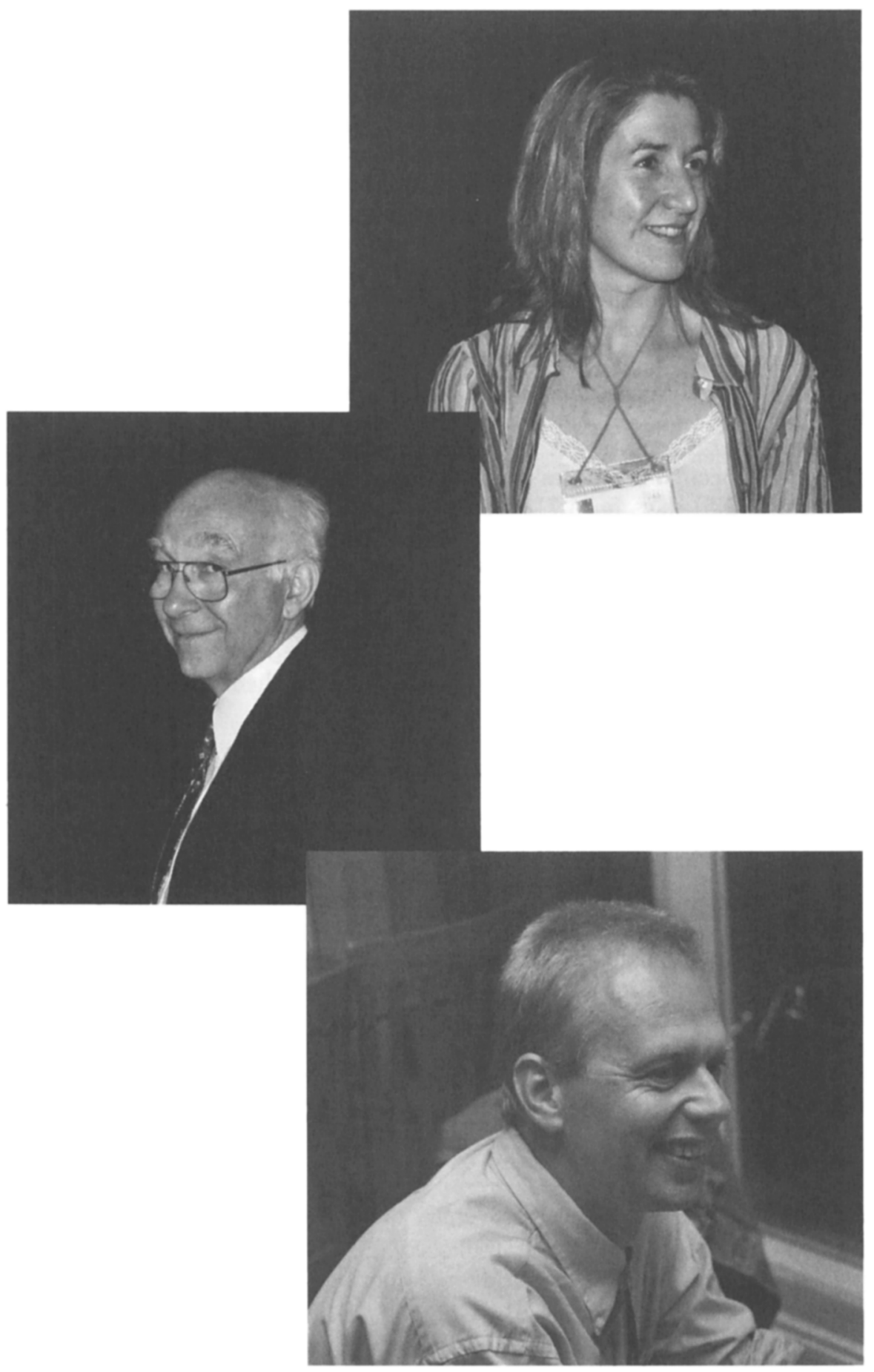

